\title{
Brn3a-Expressing Retinal Ganglion Cells Project Specifically to Thalamocortical and Collicular Visual Pathways
}

\author{
Lely A. Quina, ${ }^{1}$ Winnie Pak, ${ }^{2}$ Jason Lanier, ${ }^{1}$ Premilla Banwait, ${ }^{1}$ Kevin Gratwick, ${ }^{1}$ Ying Liu, ${ }^{1}$ Tomoko Velasquez, ${ }^{2}$ \\ Dennis D. M. 0'Leary, ${ }^{2}$ Martyn Goulding, ${ }^{2}$ and Eric E. Turner ${ }^{1}$ \\ ${ }^{1}$ Department of Psychiatry, University of California, San Diego and Veterans Affairs San Diego Healthcare System, La Jolla, California 92093-0603, and \\ ${ }^{2}$ Molecular Neurobiology Laboratory, The Salk Institute, La Jolla, California 92037
}

\begin{abstract}
Retinal ganglion cells (RGCs) innervate several specific CNS targets serving cortical and subcortical visual pathways and the entrainment of circadian rhythms. Recent studies have shown that retinal ganglion cells express specific combinations of POU- and LIM-domain transcription factors, but how these factors relate to the subsequent development of the retinofugal pathways and the functional identity of RGCs is mostly unknown. Here, we use targeted expression of an genetic axonal tracer, tau/ $\beta$-galactosidase, to examine target innervation by retinal ganglion cells expressing the POU-domain factor Brn3a. Brn3a is expressed in RGCs innervating the principal retinothalamic/retinocollicular pathway mediating cortical vision but is not expressed in RGCs of the accessory optic, pretectal, and hypothalamic pathways serving subcortical visuomotor and circadian functions. In the thalamus, Brn3a ganglion cell fibers are primarily restricted to the outer shell of the dorsal lateral geniculate, providing new evidence for the regionalization of this nucleus in rodents. Brn3a RGC axons have a relative preference for the contralateral hemisphere, but known mediators of the laterality of RGC axons are not repatterned in the absence of Brn3a. Brn3a is coexpressed extensively with the closely related factor Brn3b in the embryonic retina, and the effects of the loss of Brn3a in retinal development are not severe, suggesting partial redundancy of function in this gene class.
\end{abstract}

Key words: retina; retinal ganglion cell; lateral geniculate; accessory optic system; pretectum; superior colliculus; POU-domain; Brn3; Brn3a; Brn3b

\section{Introduction}

The output of the retina is conveyed by a population of specialized neurons that form its innermost cellular layer, the retinal ganglion cells (RGCs). All RGC axons leave the eye by a common pathway, the optic nerve. Subsequently, however, these axons must make complex pathfinding decisions that lead to the innervation of highly specific targets in the thalamus, hypothalamus, pretectum, and superior colliculus. After exiting the retina, growing RGC axons must first either cross the midline and project to the contralateral hemisphere, forming the optic chiasm, or avoid the midline and project ipsilaterally (Williams et al., 2004). Recent studies have shown that decreased expression of the $\mathrm{Zn}$ finger transcription factor Zic2 is associated with a reduced number of ipsilateral axons (Herrera et al., 2003). Conversely, RGCs expressing the LIM-homeodomain factor Islet2 project only to the contralateral side, and in the absence of Islet 2 , the extent of the ipsilateral projection is increased (Pak et al., 2004).

Progress has also been made in understanding the two-

Received July 10, 2005; revised Nov. 3, 2005; accepted Nov. 4, 2005.

This work was supported in part by Department of Veterans Affairs MERIT funding, National Institutes of Health (NIH) Awards HD33442 and MH065496 (E.E.T), and NIH Award EY07025 (D.D.M.O.). E.E.T. is a NARSAD (National Alliance for Research on Schizophrenia and Depression) Investigator. We thank Dr. Harvey Karten for extensive advice, Dr. Marla Feller for comments on this manuscript, and Drs. William Klein, Sam Pfaff, and Stephen Brown for generous gifts of reagents.

Correspondence should be addressed to Eric E. Turner, Department of Psychiatry, 0603, University of California, San Dieg0, 9500 Gilman Drive, La Jolla, CA 92093-0603. E-mail: eturner@UCSD.edu.

DOI:10.1523/JNEUROSCI.2837-05.2005

Copyright $\odot 2005$ Society for Neuroscience 0270-6474/05/2511595-10\$15.00/0 dimensional topographical mapping of the retina onto the superior colliculus (McLaughlin et al., 2003), and several classes of transcription factors are known to participate in the establishment of the retina and midbrain axes. However, in contrast to laterality decisions at the chiasm and retinocollicular mapping, little is known about how other events in RGC axon pathfinding are regulated at the transcriptional level, including the specific innervation of the SCN (suprachiasmatic nucleus), pretectal area, dorsal and ventral lateral geniculate, and accessory optic system.

We have been engaged in studies of the Brn3 class of POUhomeodomain factors in the development of the nervous system. All three members of this class, Brn3a, Brn3b, and Brn3c, are expressed in postmitotic RGCs (Xiang et al., 1995). Mice lacking Brn3b exhibit a marked loss of retinal ganglion cells and profound defects in axon growth, with only a small fraction of RGC axons extending beyond the chiasm (Gan et al., 1999; Erkman et al., 2000). In contrast, a retinal phenotype has not been described for Brn3a mutants, although loss of Brn3a expression results in severe phenotypes elsewhere in the nervous system (McEvilly et al., 1996; Xiang et al., 1996; Eng et al., 2001). The functional properties of Brn3a and Brn3b appear to be closely related, because recent experiments have shown that targeted replacement of Brn3b coding sequence with that of Brn3a rescues the developmental defects in Brn3b null retinas (Pan et al., 2005).

In the present study, we use genetically targeted expression of a tau/ $\beta$-galactosidase (tauLacZ) marker to trace the highly specific retinofugal projections of the Brn3a-expressing RGCs. 
Brn3a RGC axons avoid the suprachiasmatic nucleus, accessory optic pathway, and pretectum entirely and target very specific areas in the thalamus. Brn3a axons also project disproportionately to the contralateral hemisphere. The Brn3a null phenotype in the embryonic retina is not severe, suggesting that this factor is less critical to retinal development than Brn3b. Together, these results implicate a role for Brn3a in aspects of the genetic regulation of RGC axon guidance that have not been considered in previous studies.

\section{Materials and Methods}

Generation of mutant mice. The mice carrying a null allele of Brn3a used in the present study have been described previously (Xiang et al., 1996; Eng et al., 2001). Transgenic mice with a tauLacZ reporter allele (Mombaerts et al., 1996) targeted to the Brn3a locus were generated by recombination in embryonic stem (ES) cells. ES cells derived from 129 Sv mice were electroporated with the targeting construct (see Fig. 2) and plated on mitotically inactivated STO (SIM mouse embryo-derived thioguanine- and ouabain-resistant) feeder cells. G418 selection was begun on day 2, and colonies were picked on days 9 and 11. ES cell clones were screened by PCR demonstrating correct integration of the $3^{\prime}$ end of the transgene, and 2 of 372 clones were shown to have the expected structure by Southern hybridization using $5^{\prime}$ and $3^{\prime}$ probes. One of the correctly targeted clones was used for injection into blastocysts from C57BL/6 donors, and the resulting chimeric offspring were outbred and the progeny assayed for germline transmission of the targeted allele by PCR. Animals with the targeted transgene were then interbred with a protamine-Cre deleter strain (O'Gorman et al., 1997) to excise the neomycin resistance cassette. Mice and embryos were genotyped for the Brn3a null allele, Brn3a wildtype allele, and tauLacZ knock-in allele by PCR.

Retinal ganglion cell axon tracing. RGC axons were labeled with cholera toxin subunit B (CTB) conjugated to Alexa 594 (Invitrogen, San Diego, CA). Mice were anesthetized, and eyes were filled with $0.5 \mu \mathrm{l}$ of $2 \mathrm{mg} / \mathrm{ml} \mathrm{CTB}$ for embryonic day 18.5 (E18.5), postnatal day 10 (P10), and P30 animals or $1 \mu \mathrm{l}$ for adults. For studies performed at E18.5, pups were delivered surgically $\sim 24 \mathrm{~h}$ before normal parturition. After CTB injections, mice were returned to cages for an appropriate labeling period, then reanesthetized, perfused with $4 \%$ paraformaldehyde, and sectioned at $30 \mu \mathrm{M}$ on a cryostat. The labeling period was $8 \mathrm{~h}$ for E18.5 mice, $24 \mathrm{~h}$ for P10, $48 \mathrm{~h}$ for P30, and $72 \mathrm{~h}$ for adults. Some mice examined at P10 and P30 were anesthetized and unilaterally enucleated at P2.

Immunofluorescence and in situ hybridization. Rabbit antisera to Brn3a and Brn3b have been described previously (Fedtsova and Turner, 1995; Trieu et al., 1999). Guinea pig antisera to Brn3a were prepared against a glutathione $S$-transferase fusion protein containing an 80 amino acid fragment of mouse Brn3a containing sequences $\mathrm{N}$-terminal to the POUspecific domain that are not conserved across members of the Brn3 (POU4) gene class. All Brn3-family antisera were antigen-affinity puri-

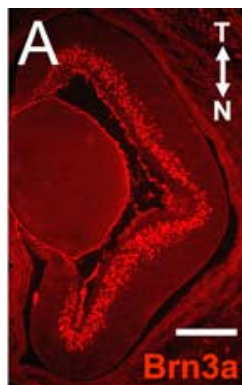

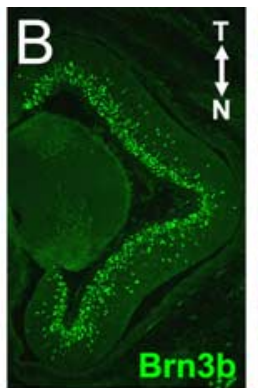
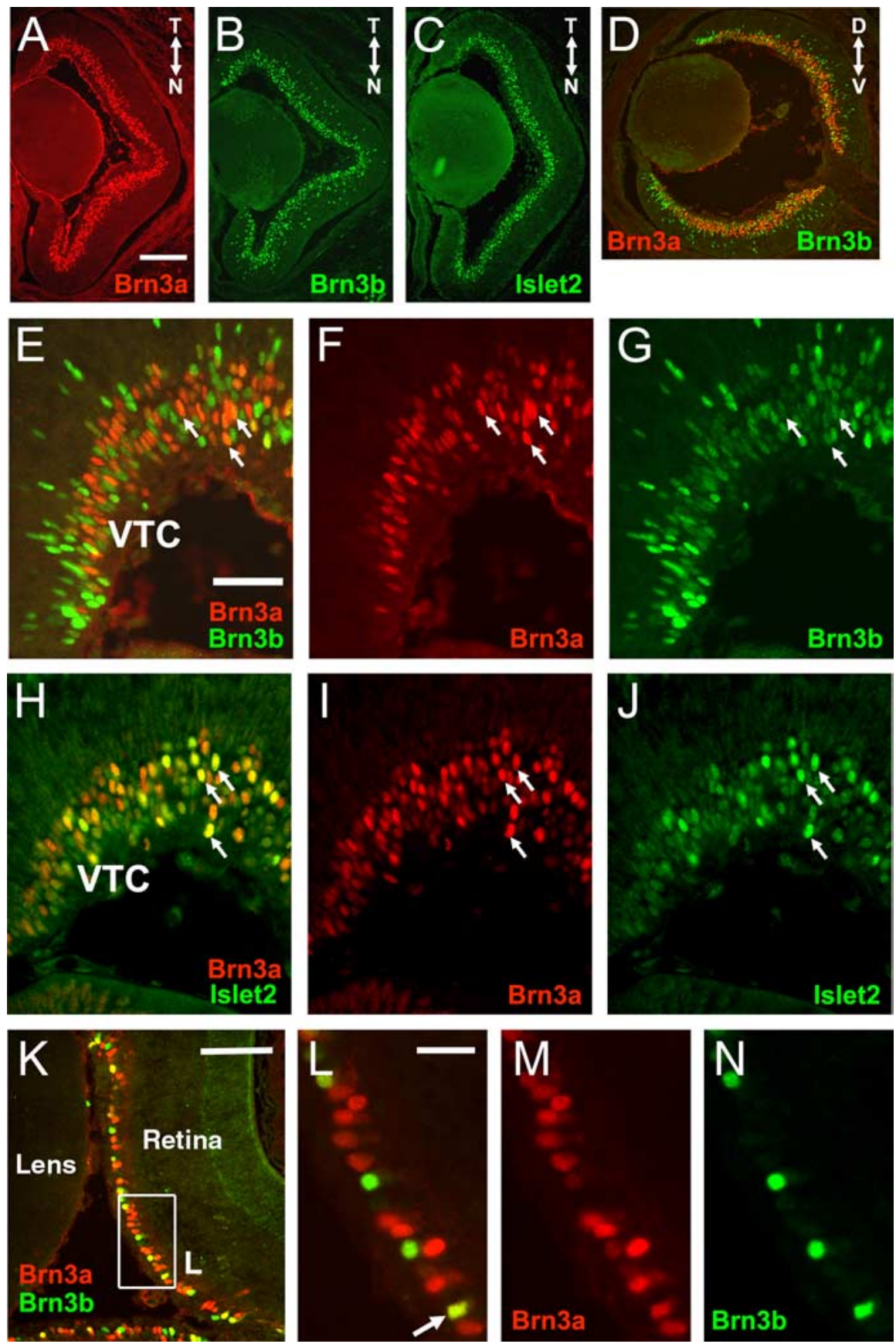

Figure 1. Expression of Islet2 and POU4-class transcription factors in the normal retina. $A-C$, Horizontal sections of the E16.5 retina, stained with primary antibodies for Brn3a, Brn3b, and Islet2, as shown. N, Nasal; T, temporal. D, Sagittal section of the E16.5 retina showing double-label immunofluorescence for Brn3a and Brn3b. D, Dorsal; V, ventral. $E-G$, Enlarged horizontal views at the same stage showing nuclear localization of Brn3a and Brn3b; nearly all RGCs strongly immunoreactive for Brn3a (arrows) also express Brn3b at low levels. $\boldsymbol{H}$-J, Enlarged views showing localization of Brn3a and Islet2; strongly Islet2-immunoreactive $\mathrm{RGCS}$ (arrows) are a subset of the Brn3a-expressing cells. $\boldsymbol{K}-\boldsymbol{N}, \mathrm{Brn} 3 \mathrm{a}$ and Brn3b expression in the P5 retina. The arrow indicates an RGC coexpressing both factors. Scale bars: (in $\boldsymbol{A}) \boldsymbol{A}-\boldsymbol{D}, 200 \mu \mathrm{m}$; (in $\boldsymbol{E}) \boldsymbol{E}-J, 50 \mu \mathrm{m} ; \boldsymbol{K}, 100 \mu \mathrm{m} ; \boldsymbol{L}, 25 \mu \mathrm{m}$.

fied before use. Guinea pig anti-Islet2 antiserum was a gift from Dr. Sam Pfaff (Salk Institute, La Jolla, CA). Rabbit anti-Zic2 antibody was a gift from Dr. Stephen Brown (Columbia University, New York, NY). Rabbit anti- $\beta$-galactosidase was obtained from 5 Prime-3Prime (Boulder, $\mathrm{CO}$ ), and goat anti- $\beta$-galactosidase was obtained from Biogenesis (Sandown, $\mathrm{NH}$ ). For all immunofluorescence studies, embryos were fixed with $4 \%$ paraformaldehyde by immersion (E16.5 and earlier) or by perfusion (E18.5 and postnatal stages). Cryosections were cut at $10-15 \mu \mathrm{m}$ for cellular localization of nuclear antigens and $30 \mu \mathrm{m}$ for tracing of RGC axons. Methods for the staining of $\beta$-galactosidase activity with 

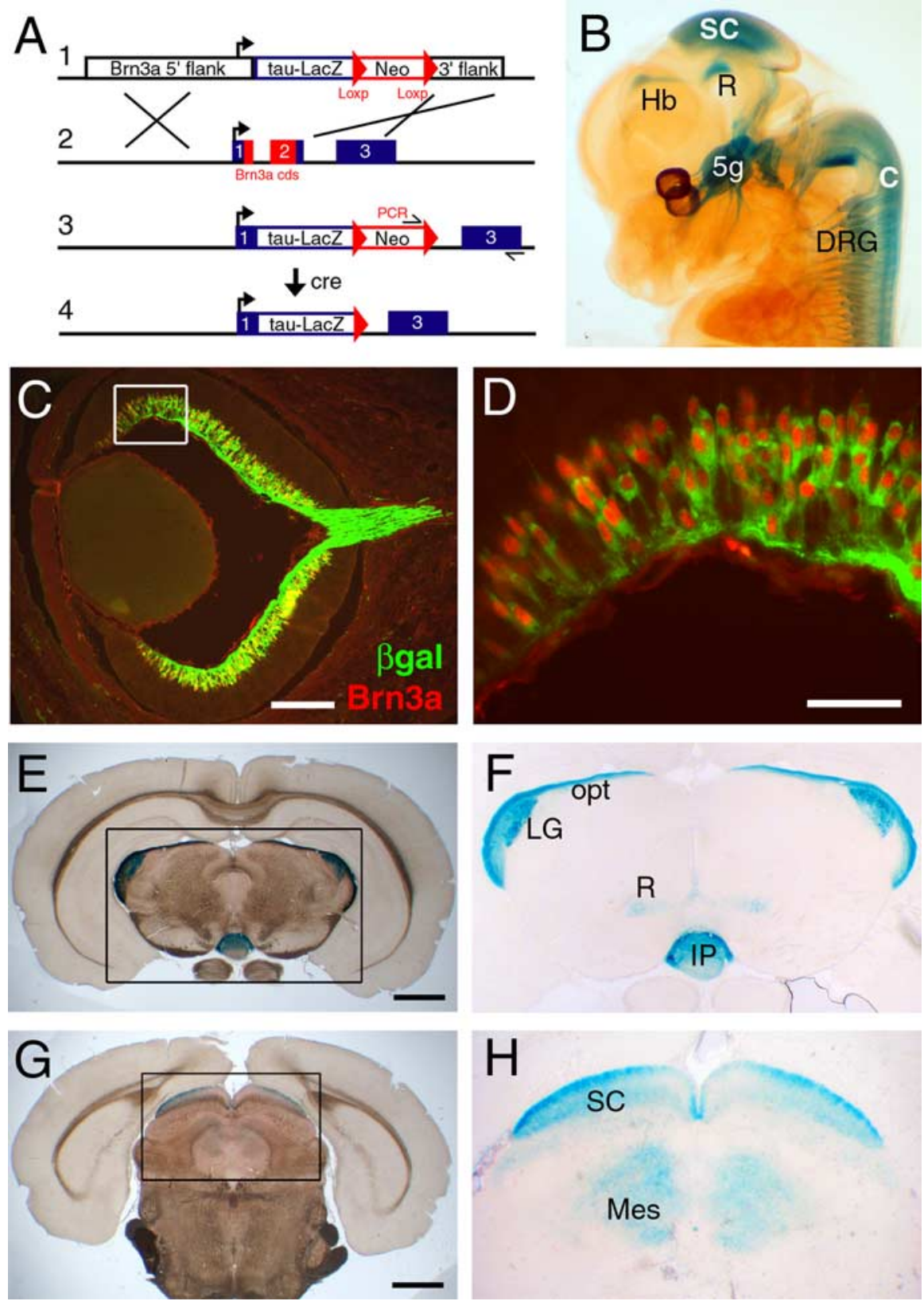

Figure 2. Targeted expression of a tauLacZ reporter integrated at the Brn3a locus. $A$, In the targeting strategy shown, integration of a taulacZ reporter replaces the entire Brn3a coding sequence. The neomycin (Neo) resistance cassette is subsequently excised by crossing with a protamine-Cre deleter line. $B$, Expression of the tauLacZ transgene replicates all known sites of Brn3a expression in an E13.5 Brn3a $a^{\text {taulac }}$ embryo. C, D, Colocalization of $\beta$-galactosidase ( $\beta$ gal) and Brn3a immunoreactivity in the retina of an E16.5 Brn3a $a^{\text {tlacZ }}$ embryo. $\boldsymbol{E}-\boldsymbol{H}$, Expression of the tauLacZ transgene in the adult brain at the level of the caudal thalamus $(\boldsymbol{E}, \boldsymbol{F})$ and at the level of the superior colliculus $(\boldsymbol{G}, \boldsymbol{H})$. $5 \mathrm{~g}$, Trigeminal ganglion; $C$, spinal cord; DRG, dorsal root ganglia; $\mathrm{Hb}$, habenula; $I \mathrm{P}$, interpeduncular nucleus; $\mathrm{LG}$, lateral geniculate; opt, optictract; Mes, mesencephalon (deep gray); $\mathrm{R}$, red nucleus; SC, superior colliculus. Scale bars: C, $200 \mu \mathrm{m} ; \boldsymbol{D}, 50 \mu \mathrm{m} ; \boldsymbol{E}, \mathbf{G}, 1 \mathrm{~mm}$.

5-bromo-4-chloro-3-indolyl- $\beta$-D-galactopyranoside (Xgal) and clearing of whole stained embryos in benzyl alcohol/benzyl benzoate have been described previously (Eng et al., 2001). For staining of $\beta$-galactosidase activity in the adult brain, brains were fixed by perfusion with $4 \%$ formaldehyde, saturated with sucrose, mounted in OCT solution, and cut frozen at $60 \mu \mathrm{m}$. Xgal staining was performed on floating sections, which were then mounted on slides for dark-field and bright-field microscopy.

Methods for non-isotopic in situ hybridization and the plasmids encoding the probes used for GATA3, AP2 $\beta$, Math3, NeuroD1, Irx1, and Irx2 have been described previously (Eng et al., 2004). The persyn cDNA probe was a gift from Dr. William Klein (M.D. Anderson Cancer Center, Houston, TX).

\section{Results}

To begin to understand the role of Brn3a in postmitotic ganglion cell development, we examined the expression patterns of Brn3a, Brn3b, and Islet 2 in the developing retina. It has been reported previously that Brn3b can first be detected in the earliest postmitotic ganglion cells at E11.5 and that Brn3a expression follows by 1-2 developmental days (Pan et al., 2005). Like Brn3a, Islet2 expression begins at E13.5, and previous work has shown that at E15.5, approximately one-third of RGCs express Islet2 (Pak et al., 2004). At E16.5, when retinofugal axons are actively growing into the CNS, Brn3a, Brn3b, and Islet2 are expressed widely in RGCs and do not exhibit marked nasotemporal or dorsoventral gradients (Fig. $1 A-D$ and data not shown) (Xiang et al., 1995). Few neurons in the ventrotemporal crescent (VTC) strongly express either Brn3a or Islet2, whereas Brn3b RGCs are abundant in this area (Fig. $1 E, H$ ). Finally, from E13.5 to E16.5, only Brn3b is expressed strongly in a population of postmitotic RGCs that are interspersed in the neuroepithelial layer, whereas Brn3a and Islet 2 are confined exclusively to the ganglion cell layer (Fig. 1E-J).

Although the global expression patterns of these three factors are similar in the E16.5 retina, they can be distinguished at the cellular level. The RGC populations most strongly expressing Brn3a and Brn $3 b$ are distinct, although almost all Brn3aexpressing cells coexpress Brn3b at a low level at this stage (Fig. 1E-G). The Brn3a and Islet 2 populations significantly overlap, and the RGCs strongly positive for Islet2 appear to be a subset of the Brn3aexpressing neurons (Fig. $1 \mathrm{H}-\mathrm{J}$ ). At P5, $\mathrm{Brn} 3 \mathrm{a}$ is the predominant POU factor, although a subset of RGCs continues to express Brn3b strongly (Fig. $1 K-N$ ). By this stage, the coexpression of $\mathrm{Brn} 3 \mathrm{a}$ and Brn3b is much less pronounced, and most of the Brn3a RGCs either do not express Brn3b or express it at undetectable levels. Together, these results indicate that Brn3a, Brn3b, and Islet2 may function combinatorially and sequentially to specify the fates of classes of RGCs that are distributed throughout most of the retina.

To better understand the role of Brn3a in the development of the retina and other regions of the CNS in which it is expressed, we used targeted deletion of Brn3a in embryonic stem cells combined with the introduction of a bovine tau/ $\beta$-galactosidase fusion gene to create a strain of reporter mice $\left(B r n 3 a^{\text {tLacZ }}\right)$. In the targeting strategy used, the tauLacZ transgene, combined with a neomycin resistance cassette, replaced the entire Brn3a coding sequence and a small intron within the Brn3a open reading frame (Fig. 2A). Founder mice with germline transmission of the 
$B r n 3 a^{t L a c Z}$ allele were then interbred with mice containing a protamine-Cre transgene, resulting in excision of the neomycin resistance cassette.

At E13.5, the expression of the Brn3a ${ }^{t L a c Z}$ transgene (Fig. 2B) correctly replicates the expression of Brn3a in all areas of the CNS, including the habenula, red nucleus, superior colliculus, inferior olivary nucleus, and spinal cord (Turner et al., 1994; Xiang et al., 1996; Trieu et al., 1999). In the peripheral nervous system, the transgene is correctly expressed in the trigeminal ganglion, components of the vestibulocochlear and IX/X ganglion complexes, and the dorsal root ganglia (Fedtsova and Turner, 1995; Eng et al., 2001). Thus, it is likely that in addition to the retina, $B r n 3 a^{t L a c Z}$ transgenic mice will be useful for developmental studies of several CNS areas and the peripheral sensory system. Axons of the Brn3a-expressing RGCs are not sufficiently developed to be visualized in whole mounts at E13.5, but $\beta$-galactosidase is detectable by immunofluorescence in the ganglion cell layer of the retina.

In accordance with the relatively late differentiation of Brn3a RGCs (compared with those expressing Brn3b), the retinal axons expressing the $B r n 3 a^{t L a c Z}$ transgene follow established tracts from the retina to the chiasm, which are evident by immunofluorescence for neurofilament-160 1-2 d before the appearance of the $B r n 3 a^{t L a c Z}$ fibers (data not shown). By E16.5, the tauLacZ marker is coexpressed strongly with Brn3a in numerous RGCs (Fig. 2C,D), and the Brn3a RGC axons have exited the retina (Fig. 2C) and reached the optic chiasm (data not shown).

Brn3a persists in the mature nervous system (Trieu et al., 1999), and the $B r n 3 a^{t L a c Z}$ transgene allows visualization
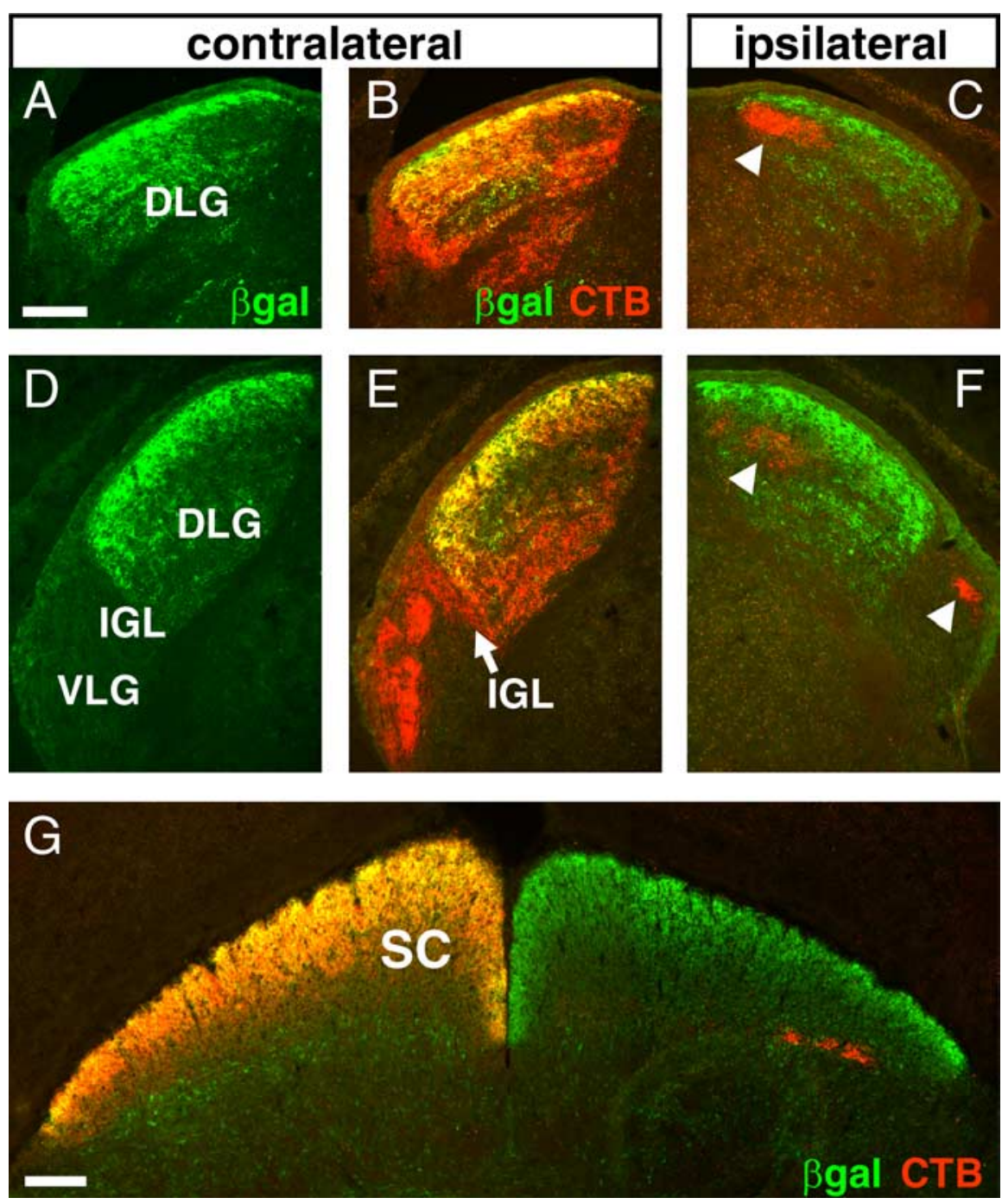

Figure 3. Innervation of the adult lateral geniculate and superior colliculus by Brn3a RGCs. Alexa-594 CTB was injected unilaterally, and animals were killed after $3 \mathrm{~d}$. Expression of the tauLacZ transgene was visualized with immunofluorescence for $\beta$-galactosidase. Brn3a RGC axons predominantly innervate the superficial layer of the DLG and are absent from the principal part of the VLG and all ipsilateral $L G$ fibers. $\boldsymbol{A}-\boldsymbol{C}$, Retinal projections to the rostral lateral geniculate. $\boldsymbol{D}-\boldsymbol{F}$, Innervation of the caudal lateral geniculate. $\boldsymbol{G}$, Retinal fibers innervating the superior colliculus. The arrowheads in $\boldsymbol{C}$ and $\boldsymbol{F}$ indicate ipsilateral fibers, which are devoid of $\beta$-galactosidase immunoreactivity. $\beta$ gal, $\beta$-Galactosidase; SC, superior colliculus. Scale bars: (in $A, G) 100 \mu \mathrm{m}$. of the axon projections of Brn3a-expressing neurons in the adult CNS and peripheral nervous system (Fig. $2 E-H$ ). In the area of the optic tracts and retinoreceptive thalamic nuclei (Fig. $2 F$ ), Brn3a protein is not expressed, and the tauLacZ marker labels only retinal projections and their termini. In the midbrain (Fig. $2 H)$, Brn3a is expressed in a laminar pattern in the superior colliculus and mesencephalic central gray, and $\beta$-galactosidase activity stains both these intrinsic midbrain neurons and the projections of Brn3a RGCs innervating the superior colliculus.

To better understand the role of Brn3a in retinal development, we next performed detailed studies of the retinofugal pathways in $B r n 3 a^{\text {tLacZ }}$ heterozygous mice. Although these studies were performed in mice possessing only one functional Brn3a allele, no phenotype has ever been detected in any part of the nervous system in Brn3a heterozygotes. Furthermore, in recent work, we have demonstrated that in the sensory peripheral nervous system, the Brn3a heterozygote phenotype is effectively suppressed by gene dosage compensation (Trieu et al., 2003; Eng et al., 2004).
Thus, it is likely that the heterozygous mice in these studies are phenotypically wild type.

The retinofugal axons of intact adult mice carrying the $B r n 3 a^{\text {tLacZ }}$ allele were examined by the injection of one eye with fluorescently labeled CTB. After $72 \mathrm{~h}$, the animals were killed and examined by fluorescence microscopy for $\beta$-galactosidase expression and localization of the CTB marker. Examination of the retinofugal axons of the adult $B r n 3 a^{t L a c Z}$ mouse reveals remarkable specificity in the projections of the Brn3a RGCs (Fig. 3). Numerous Brn3a RGC fibers terminate in the dorsal lateral geniculate nucleus (DLG) and show a strong preference for the superficial layers of the nucleus. In contrast, the principal part of ventral lateral geniculate nucleus (VLG) and the intergeniculate leaflet (IGL) are devoid of $\beta$-galactosidase-immunoreactive fibers, although a few labeled fibers appear to terminate in the most superficial layer overlying the VLG (see Figs. $3 D, E, 5$ ). In the DLG, ipsilateral projections terminate in a centrally located eyespecific layer (Godement et al., 1984). No $\beta$-galactosidase immunoreactivity is detectable in the CTB-labeled axon termini in the 

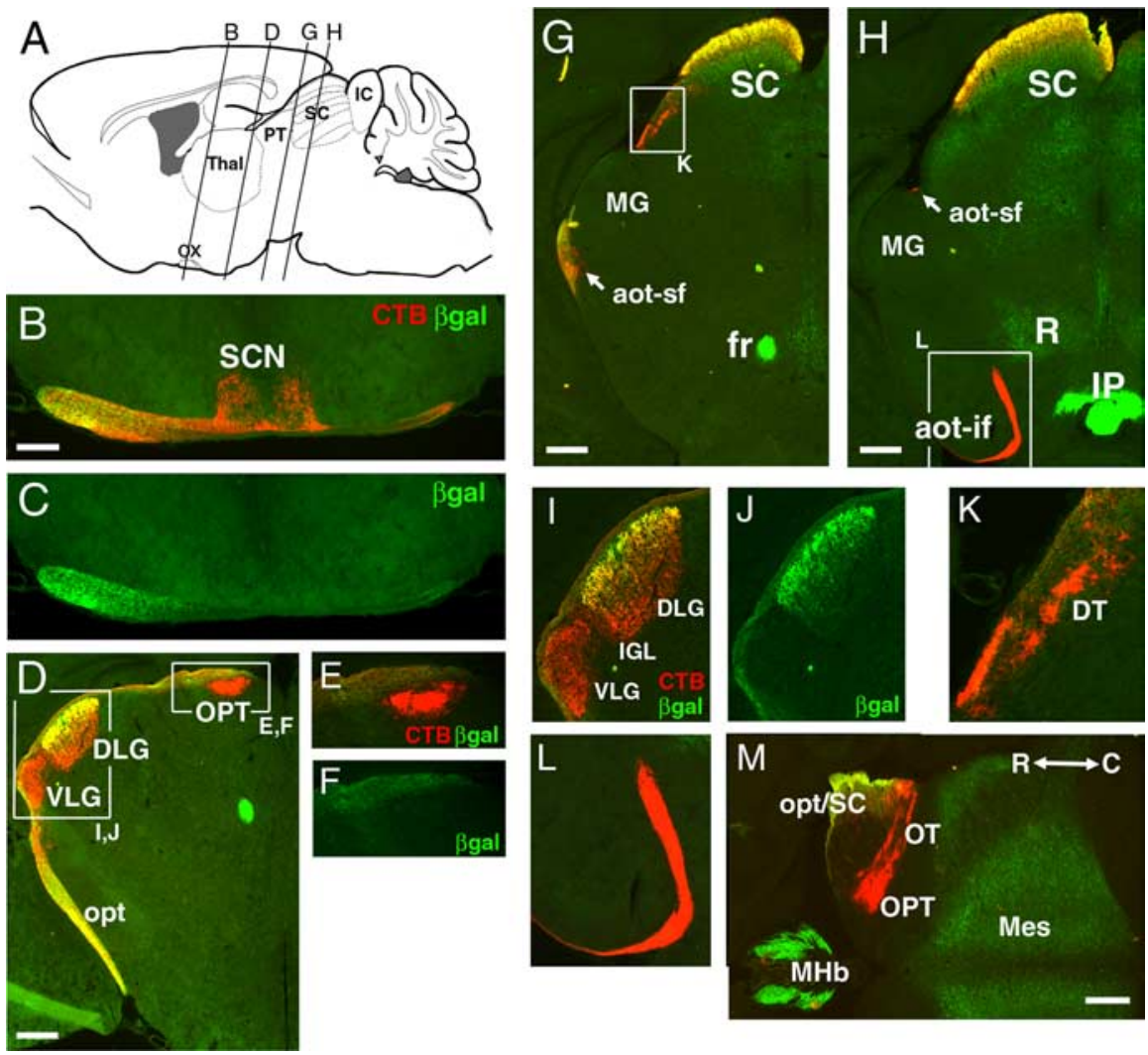

Figure 4. Restricted targets of Brn3a RGCs in the thalamus and midbrain. ABrn3a $a^{\text {tLacZ }}$ mouse was enucleated unilaterally at $P 2$, and the remaining eye was injected with CTB $2 \mathrm{~d}$ before the mice were killed at P30. Except for $\boldsymbol{B}$ and $\boldsymbol{C}$, only the contralateral side is shown. $\boldsymbol{A}$, Planes of coronal section for views $\boldsymbol{A}-\boldsymbol{L}$. $\boldsymbol{B}, \boldsymbol{C}$, Section immediately posterior to optic chiasm shows that retinal projection to the $S C N$ is devoid of $\beta$-galactosidase immunoreactivity. $D-F, I, J$, Section at level of the caudal lateral geniculate and pretectum. The pretectal nuclei and the principal part of the VLG show no $\beta$-galactosidase immunoreactivity. $\boldsymbol{G}, \boldsymbol{K}$, Section through the rostral superior colliculus. $\boldsymbol{H}, \boldsymbol{L}$, Section near the middle of the superior colliculus, showing the accessory optic tract, which does not exhibit $\beta$-galactosidase immunoreactivity. $M$, Horizontal section through the midbrain and pretectal area. Aot, Accessory optic tract (sf, superior fasciculus; if, inferior fasciculus); DT, dorsal terminal nucleus of the accessory optic tract; fr, fasciculus retroflexus; IC, inferior colliculus; IP, interpeduncular nucleus; Mes, mesencephalon (deep layers of SC and central gray); $\mathrm{MHb}$, medial habenula; MG, medial geniculate; opt, optic tract; OPT, olivary pretectal nucleus; 0T, nucleus of the optic tract; $0 \mathrm{x}$, optic chiasm; PT, pretectum; $R$, red nucleus; SC, superior colliculus; Thal, thalamus; $\beta$ gal, $\beta$-galactosidase; $R$, rostral; $C$, caudal. Where possible, nomenclature is adopted from Paxinos and Franklin (2001). Scale bars: $\boldsymbol{B}, 200 \mu \mathrm{m} ; \boldsymbol{D}, \boldsymbol{G}, \boldsymbol{H}, \boldsymbol{M}, 400 \mu \mathrm{m}$.

main ipsilateral layers in either the DLG or the VLG (Fig. 3C,F), demonstrating the contralateral preference of the Brn3a RGCs. In the superior colliculus, the LacZ marker labels both the afferent Brn3a RGC fibers and the axons of intrinsic Brn3a-expressing neurons in the deeper lamina. In contrast to the lateral geniculate, the projections of Brn3a RGCs to the superior colliculus appear to be uniformly distributed relative to the CTB label in all three axes (Fig. $3 G$ ).

To determine the lateralization of the projections of Brn3a RGCs originating from a single eye, not possible with LacZ staining alone, we examined Brn3a $a^{t L a C Z}$ mice that were enucleated unilaterally at $\mathrm{P} 2$, followed by CTB injection into the remaining eye at P9 or P28, and killed at P10 or P30. As shown in Figure 4, the projections of Brn3a RGC fibers to the lateral geniculate at P30 were strongly localized to the superficial region of the DLG, very similar to the pattern seen in adult animals. Projections to the superior colliculus also resembled the pattern observed in adults. In contrast to these pathways, Brn3a RGC axons were excluded from several of the major retinofugal projections. No $\beta$-galactosidase immunoreactivity was detected in the retinal fibers innervating the suprachiasmatic nucleus (Fig. $4 B, C$ ), the
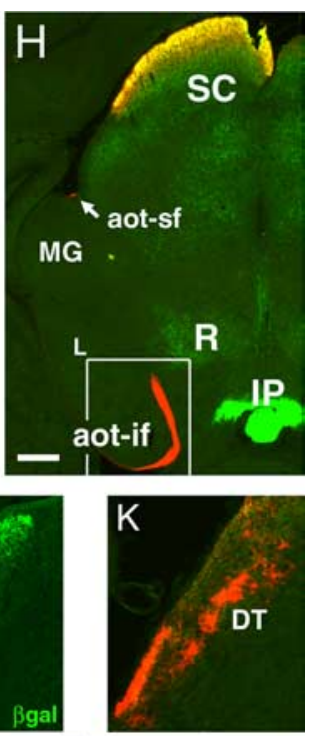

pretectal nuclei (Fig. $4 D-F, M$ ), or the accessory optic system (Fig. $4 G, H, K, L$ ).

These studies of enucleated P30 $B r n 3 a^{\text {tLac } Z}$ mice confirmed the observation made in adult animals (Fig. 3) that fibers originating from Brn3a RGCs could not be detected in the regions of the DLG or VLG where most ipsilateral fibers have been shown to terminate in previous studies. However, coronal sections through the ascending optic tracts of a unilaterally enucleated P30 Brn3a $a^{\text {tLacZ }}$ mouse (Fig. $5 A-C$ ) showed that some $\beta$-galactosidase expression is present on the ipsilateral side. To assess the relative extent of the ipsilateral projection of the Brn3a RGC axons, we used quantitative immunofluorescence. For these assays, three to five sections from each of four unilaterally enucleated animals were examined, including two at $\mathrm{P} 10$ and two at $\mathrm{P} 30$. The ratio of the $\beta$-galactosidase/CTB immunofluorescence signal on the contralateral side, which constitutes $>95 \%$ of the total fluorescence, was set to an arbitrary value of 1 and used to normalize the ipsilateral signal in each section. Although some variability in the extent of the ipsilateral projection was observed between specimens (Fig. $5 D$ ), Brn3a RGC axons displayed a clear preference for the contralateral hemisphere, and the average normalized ratio of ipsilateral $\beta$-galactosidase/CTB signal for all samples was $\sim 60 \%$ of that measured on the contralateral side.

Given that a small population of Brn3a RGC axons project ipsilaterally in unilaterally enucleated mice but do not innervate the characteristic ipsilateral target fields of the DLG, we followed the ascending ipsilateral optic tract in serial sections to locate the termini of these axons. $\beta$-Galactosidase-immunoreactive fibers could be detected in the ascending ipsilateral optic tract to the level of the VLG, where they appeared to terminate in a very superficial layer of this structure (Fig. $5 E-G$ ) but could not be detected in the more dorsal part of the optic tract which continues to the DLG and superior colliculus.

To further determine whether a normal subpopulation of Brn3a RGCs project to the ipsilateral VLG, we examined this nucleus in intact adult animals, also labeled unilaterally by injection of CTB. In contrast to the animals enucleated at P2, no $\beta$-galactosidase immunoreactivity colocalized with the termini of ipsilateral projections in the VLG (Fig. $5 H-M$ ) in these specimens, and the mature projections of the Brn3a RGCs to the VLG thus appear to be entirely contralateral. Together, these results suggest that a small number of Brn3a RGCs make an initial projection to the ipsilateral VLG, which regresses in postnatal development if the contralateral projection is present but persists abnormally in unilaterally enucleated animals.

Next, we examined the effect of the loss of Brn3a on RGC axon growth and retinal gene expression. To maintain a constant dosage of the tauLacZ reporter gene in heterozygous and knock-out 
mice in the tract-tracing studies, we interbred $B r n 3 a^{t L a c Z}$ mice with a previously characterized null mutant (Xiang et al., 1996; Eng et al., 2004) to produce offspring with the genotypes Brn3at ${ }^{\text {LacZ/+ }}$ (control) and Brn3a $a^{\text {tLacZ/- }}$ (knock-out). Unfortunately, the analysis of the development of the retinofugal connections in these mice is limited by the neonatal death of the Brn3a null mutants and the relatively late development of the projections of the Brn3a RGCs. However, several informative studies were conducted late in embryogenesis.

Examination of the optic chiasm and tracts in whole-mount E16.5 embryos revealed no apparent differences between $\mathrm{Brn} 3 \mathrm{a}^{\text {tLacZ/+}}$ and Brn $3 a^{\text {tLacZ/- }}$ mice (Fig. $6 A, B)$, in contrast to the marked defects seen in the chiasm of embryos lacking Brn3b (Erkman et al., 2000). Postnatal Brn3a null mice showed very limited survival and could not be reliably kept long enough to permit labeling of the retinofugal projections. For this reason, embryos were delivered surgically at E18.5, $1 \mathrm{~d}$ before normal parturition, to allow unilateral labeling of the optic pathways by retinal injection of Alexa-594 CTB. More than $50 \%$ of the injected Brn $3 a^{t L a c Z /-}$ pups delivered at this stage survived for the $8 \mathrm{~h}$ period of labeling. CTB labeling revealed retinal fibers in the ascending optic tracts and the DLG and VLG target fields in these mice (Fig. $6 C, D$ ), but $\beta$-galactosidaseimmunoreactive fibers originating in the Brn3a RGCs remained mostly in the tracts, running tangential to the DLG, and rarely penetrated the nucleus in either heterozygous or knock-out animals. Examination of the superior colliculus revealed that the axons of Brn3a RGCs do not reach this structure in significant numbers by E18.5 (Fig. 6E-H).

The observation that Brn3a fibers do not penetrate the LGN at E18.5 is entirely consistent with previous observations on the developmental timing of RGC differentiation and the retinogeniculate projections. Postmitotic RGCs are first observed at E11.5, but a substantial population of Brn3a-expressing RGCs does not appear until E13.5, a delay of 2 developmental days. Previous studies have shown that the earliest retinal axons reach the vicinity of the LGN at E16 (Godement et al., 1984) but run mainly tangential to the nucleus at that stage, whereas by E18, more axons run lateromedially, consistent with the CTB labeled axons in this study. Thus, the observation that Brn3a RGC axons have arrived in the vicinity of the lateral geniculate at E18.5 but do not penetrate the nucleus is entirely consistent with a $1-2 \mathrm{~d}$ lag in the developmental program of Brn3a RGCs relative to the earliest retinal neurons.

We next examined the retinas of Brn3a null mice for the effects on the expression of other transcription factors expressed in postmitotic RGCs. Previous work has shown that Brn3a and Brn3c expression is significantly diminished in the Brn3b knock-
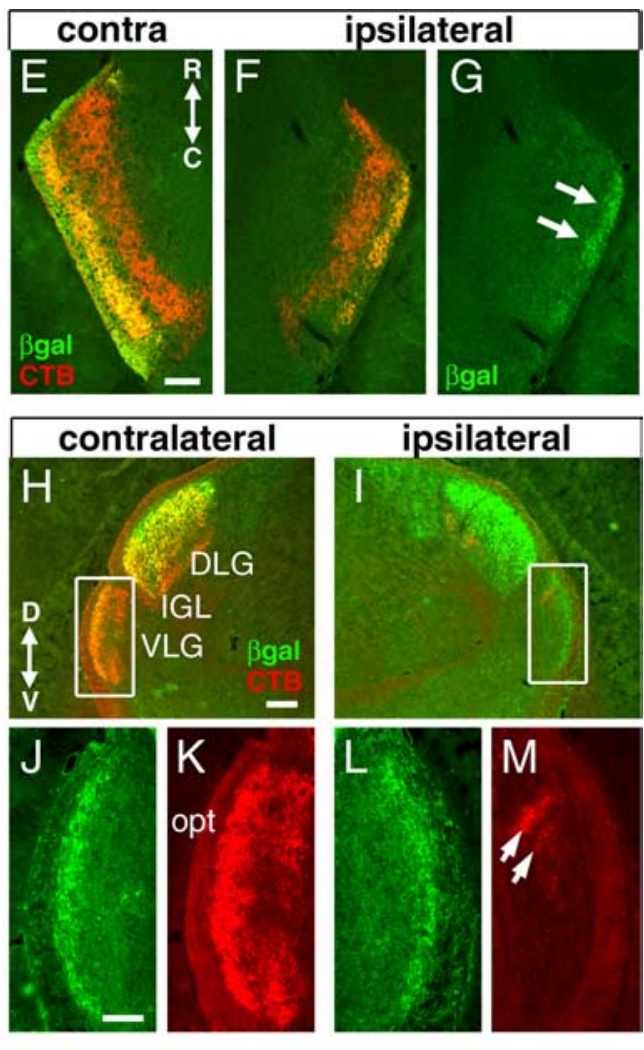

Figure 5. Laterality of Brn3a RGC axon projections. In $\boldsymbol{E}-\mathbf{G}$, animals were enucleated unilaterally at $P 2$, injected with CTB in the vitreous space at $\mathrm{P} 9$ or $\mathrm{P} 28$, and killed at $\mathrm{P} 10$ or P30. A-C, Coronal section of a P30 brain showing the ascending optic tracts. The arrow indicates ipsilateral projection to the DLG. $\boldsymbol{D}$, Quantitative immunofluorescence of the relative $\beta$-galactosidase immunoto five sections were analyzed for each condition, and the mean \pm SD is shown. Differences between the contralateral and ( (arrows) at P30. The overall size of the ipsilateral VLG is reduced because of loss of the contralateral projection after enucleation. 政 of $\beta$-galactosidase and CTB on the ipsilateral side. In adult animals, the overlying optic tract $(\boldsymbol{K})$ does not label well with $\beta$ gal, $\beta$-Galactosidase; contra, contralateral; ipsi, ipsilateral; $D$, dorsal; $V$, ventral; $R$, rostral, $C$, caudal; Hb, habenula; opt, optic tract. Scale bars: (in $\boldsymbol{B}) \boldsymbol{A}-\boldsymbol{C}, 400 \mu \mathrm{m}$; (in $\boldsymbol{E}$ ) $\boldsymbol{E}-\mathbf{G}, 100 \mu \mathrm{m}$; (in $\boldsymbol{H}$ ) $\boldsymbol{H}, \boldsymbol{I}, 200 \mu \mathrm{m}$; (in J) $\boldsymbol{J}-\boldsymbol{M}, 100 \mu \mathrm{M}$.

out retina (Gan et al., 1996). However, the converse does not appear to be true, because Brn3b expression appeared unchanged at E16.5 in retinas of mice lacking Brn3a (Fig. 7A). Consistent with the weak expression of Brn3b observed in nearly all Brn3a neurons at E16.5, Brn3b was detected in the nuclei of $\beta$-galactosidase-immunoreactive RGCs in $\mathrm{Brn} 3 \mathrm{a}^{\text {tLacZ/- }}$ retinas, indicating that Brn3b expression persists in RGCs that would normally coexpress Brn3a (Fig. 7B). Islet2 also showed no marked change in the Brn $3 a^{t L a c Z /-}$ retina, and the expression of Islet2 was maintained in RGCs that would normally express Brn3a (Fig. 7C,D).

Expression of the $\mathrm{Zn}$-finger protein $\mathrm{Zic} 2$ is associated with ipsilaterally projecting RGCs, and the domain of Zic2 expression in the ventrotemporal crescent expands in the absence of Islet2 (Pak et al., 2004). In wild-type E16.5 embryos, strongly positive Brn3a neurons were not often observed in the VTC, and Zic2 and Brn3a were rarely coexpressed (Fig. 7E). However, in contrast to Islet2 knock-outs, Zic2 expression did not expand into the Brn3a expression domain in Brn3a $a^{t L a c Z /-}$ embryos (Fig. 7F, G).

Previous expression studies using arrays and subtractive 


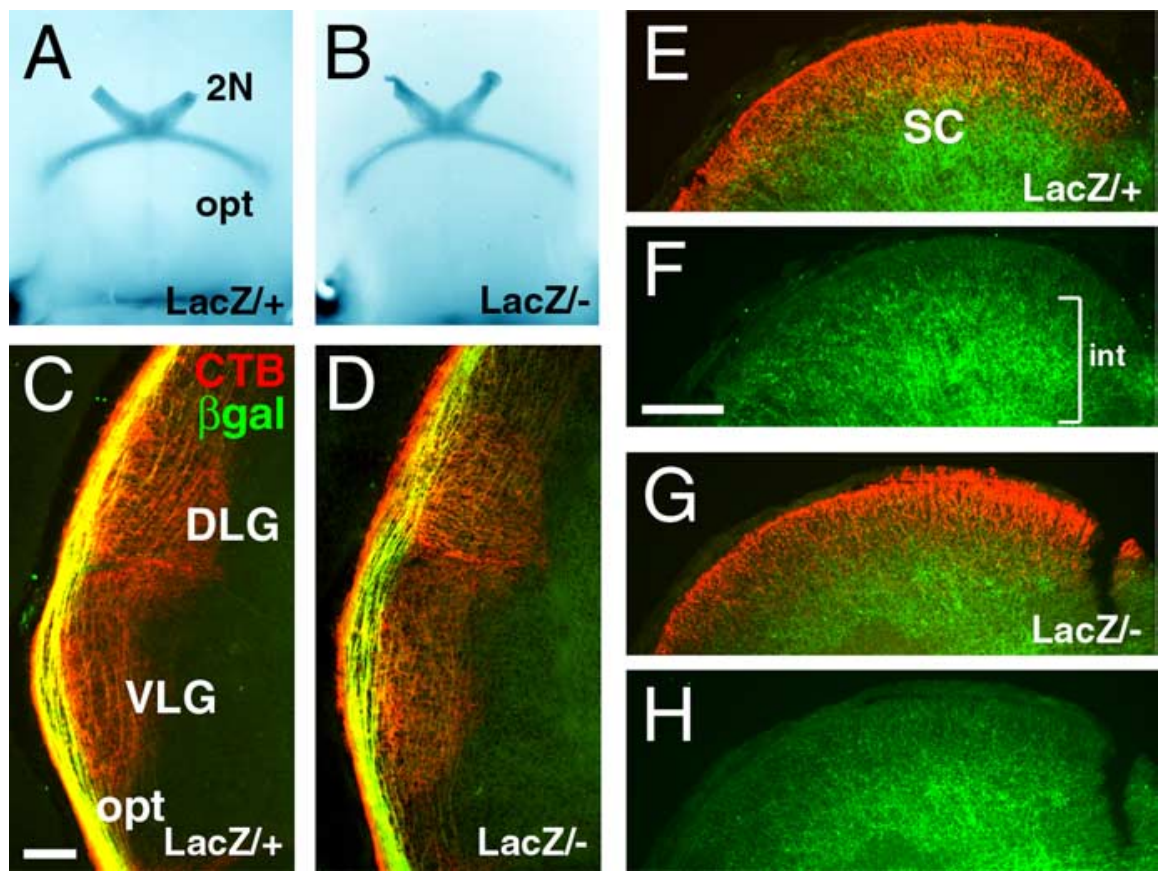

Figure 6. Development of the retinothalamic/retinocollicular tracts in Brn3a null mice. $A, B, X g a l$ staining of the optic chiasm at E16.5 in whole-brain preparations. $\boldsymbol{C}-\boldsymbol{G}$, Viable E18.5 embryos were injected with CTB unilaterally and killed after $8 \mathrm{~h}$. $\boldsymbol{C}, \boldsymbol{D}$, CTB-labeled retinal axons can be seen in the lateral geniculate, but $\beta$-galactosidase-immunoreactive fibers remain mostly in or parallel to the optic tract. $\boldsymbol{E}-\boldsymbol{H}$, In the E18.5 midbrain, extensive labeling of intrinsic (int) midbrain Brn3a neurons is observed in deep layers of the superior colliculus ( $\boldsymbol{F}$, bracket), but few axons of the Brn3a RGCs have reached the superior colliculus at this stage in either Brn3a $a^{\text {tLacZ/+ }}(\boldsymbol{E}, \boldsymbol{F})$ or Brn3a $a^{\text {tLacZ/- }}(\boldsymbol{G}, \boldsymbol{H})$ embryos. 2N, Optic nerve; opt, optic tract; SC, superior colliculus; $\beta$ gal, $\beta$-galactosidase. Scale bars: (in C) $\boldsymbol{C}-\boldsymbol{D}, 100 \mu \mathrm{m}$; (in F) $\boldsymbol{E}-\boldsymbol{H}, 200 \mu \mathrm{m}$.

methods have revealed regulatory targets for Brn3a in the trigeminal ganglion (Eng et al., 2004) and Brn3b in the retina (Erkman et al., 2000; Mu et al., 2004). A subset of these targets was examined in Brn3a $\mathrm{a}^{-1-}$ retinas at E14.5 (Fig. 8). Three transcription factors that show markedly increased expression in the cranial sensory ganglia of Brn3a knock-out mice, but are not reported as retinal targets of Brn3b, include GATA3, AP2 $\beta$, and NeuroD1. GATA3 (Fig. $8 A, B$ ) was not detected in the retinas of either control or Brn3a knock-out mice. AP $2 \beta$, for which a role in retinal development has not been described, was expressed at the border between the neuroepithelial layer and the postmitotic layer but was also unchanged in the in Brn3a $\mathrm{a}^{-1-}$ retinas (Fig. $8 C, D$ ). NeuroD1, which has a known role in retinal neurogenesis (Pennesi et al., 2003), was strongly expressed in the retinal neuroepithelium of Brn3a wild-type and knock-out mice at E14.5 and also showed no change in the absence of Brn3a (Fig. $8 E, F$ ).

The most decreased transcript in previous studies of the Brn3b knock-out retina is persyn/synuclin- $\gamma$ (Mu et al., 2004), which may play a role in neurofilament assembly (Buchman et al., 1998). Persyn mRNA is also significantly decreased in array assays of the trigeminal ganglia of Brn3a null mice (Eng et al., 2004). However, no change in persyn expression could be detected in the retina in the absence of Brn3a (Fig. 8G-J). Another set of regulatory targets in common between Brn3a in the sensory ganglia and Brn3b in the retina are the Irx family of homeodomain factors. However, Irx2 (Mu et al., 2004) and Irx6 (Erkman et al., 2000) are decreased in the Brn3b knock-out retina, whereas Irx 1 and Irx 2 are both increased in the trigeminal ganglion in the absence of Brn3a. More consistent with the previous data for Brn3a in the sensory system, Irx1 was moderately increased in E14.5 retinas lacking Brn3a, whereas Irx2 was not significantly changed (Fig. $8 \mathrm{~K}-\mathrm{N}$ ). Finally, to verify our hypothesis that Brn3a does not regulate axial patterning in the retina, we examined the expression of EphB1 and EphB3, and as expected, neither transcript was significantly changed in the absence of Brn3a (data not shown).

\section{Discussion}

Although the axons of all retinal ganglion cells exit the retina together via the optic nerve, they must subsequently make several pathfinding choices leading to the innervation of distinct targets in the hypothalamus, thalamus, pretectum, and superior colliculus (Fig. S1, available at www.jneurosci.org as supplemental material). At each of these early choice points that establish the major retinofugal pathways, the projections of Brn3a RGCs execute characteristic decisions. At the first major choice point, the optic chiasm, Brn3a RGC axons exhibit a relative preference for the contralateral hemisphere. Brn3a RGCs are never observed to project to the well characterized ipsilateral layer of the DLG (Godement et al., 1984). Although evidence from animals enucleated shortly after birth suggests that a small cohort of Brn3a RGCs initially make an ipsilateral projection to a superficial layer of the VLG, the termini of Brn3a RGCs do not colocalize with ipsilateral fibers in the VLG of adult animals, suggesting that any ipsilateral projections of Brn3a RGCs to this region regress during postnatal development.

Although Brn3a is associated primarily with contralaterally projecting RGCs, it is less likely to be a direct mediator of laterality than either the Zn-finger transcription factor Zic2 or the LIM-homeodomain protein Islet2. Zic2 is specifically expressed in the ventrotemporal crescent of the retina when RGC axons reach the chiasm, and decreased Zic2 expression is associated with a reduced number of ipsilateral axons (Herrera et al., 2003). Conversely, Islet 2 is widely expressed in the RGC layer at the time of the midline crossing decision but is reduced in the VTC and is not coexpressed with Zic2. Islet2-expressing RGCs project only to the contralateral side, and in the absence of Islet 2 , Zic2 expression and the extent of ipsilateral projection are increased. Zic2 and Islet2 expression appear unchanged in mice lacking Brn3a, suggesting that Brn3a does not directly interact with this pathway for determining laterality.

Distal to the chiasm, Brn3a RGC axons are excluded from the retinohypothalamic and accessory optic tracts. The SCN is innervated by intrinsically photosensitive melanopsin-expressing retinal ganglion cells mediating circadian responses, which also innervate the IGL (Hattar et al., 2002). Brn3a RGC axons are absent in both the SCN and IGL, and clearly this specialized population of RGCs do not express Brn3a. The accessory optic pathway and its associated nuclei mediate motion detection and optokinetic movements in all vertebrate species (Simpson, 1984). The absence of Brn3a in accessory optic RGCs suggests that it regulates factors that repel axons at the accessory optic choice points, or 

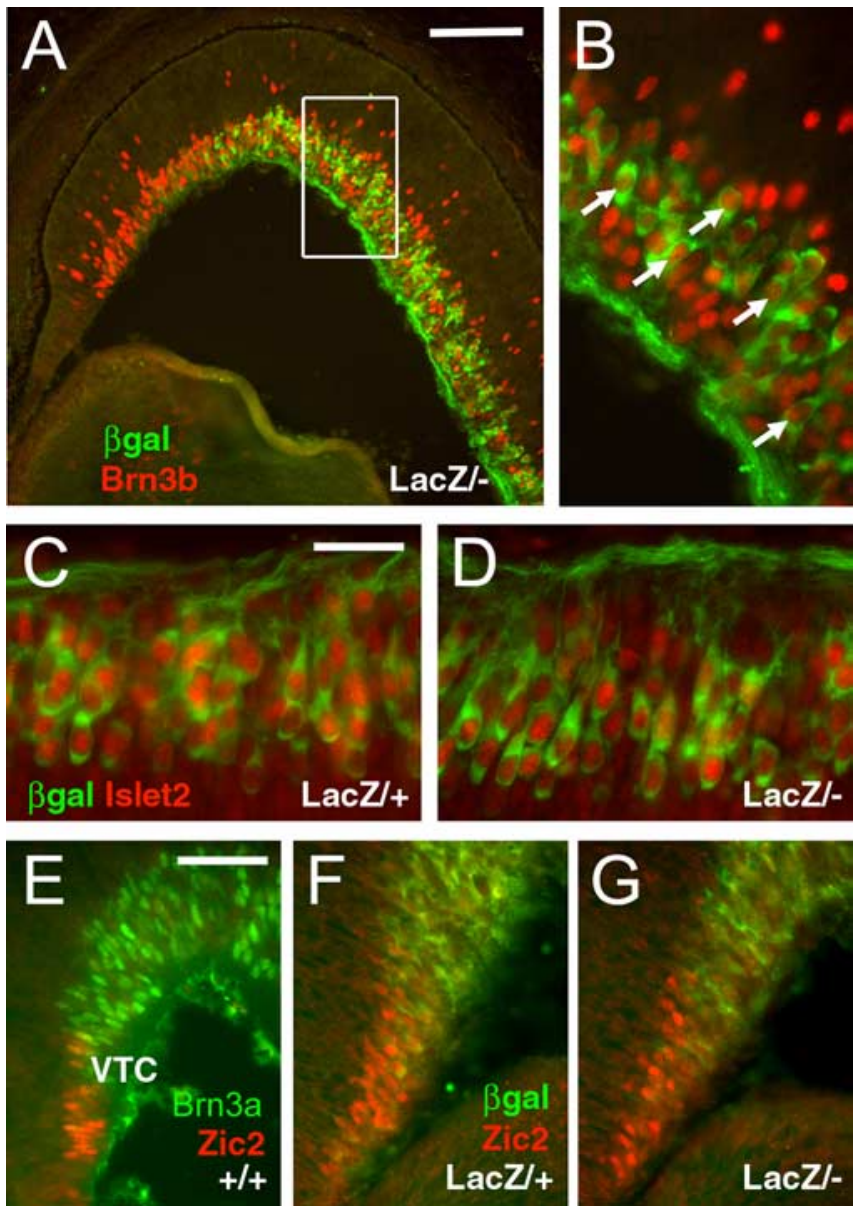

Figure 7. Transcription factor expression at E16.5 in the Brn3a knock-out retina. $A, B, B r n 3 b$ is expressed in RGCs normally fated to express Brn3a, as marked by expression of the tauLacZ transgene. C, D, Islet2 is similarly expressed in Brn3atlacZ/+ and Brn3atlacz/- mice and colocalizes with tauLaCZ in a subset of Brn3a RGCs. E, Brn3a and Zic2 are expressed in nearly nonoverlapping domains in the wild-type retina. $F, G, Z i c 2$ does not expand into the Brn3a expression domain in Brn3atlacZ/- mice. $\beta$ gal, $\beta$-Galactosidase. Scale bars: $A, 100 \mu \mathrm{m}$; (in C) C, D, 25 $\mu \mathrm{m}$; (in $\boldsymbol{E}) \boldsymbol{E}-\mathbf{G}, 50 \mu \mathrm{m}$.

conversely, maintain the integrity of the retinocollicular optic tract.

Brn3a RGC axons also execute specific pathfinding decisions at the level of the thalamus and midbrain. In carnivores and primates, the DLG exhibits a morphologically laminated structure, and in the ferret, specific layers are associated with the terminal fields of "on-center" and "off-center" RGCs (Sanes and Yamagata, 1999). In rats and mice, however, the lamination of this structure is much less obvious. It has been divided into a dorsolateral "shell," which receives only contralateral input, and a "core," which receives bilateral inputs (Reese, 1988), and the postnatal segregation of ipsilateral fibers into an eye-specific layer within the core is well known (Godement et al., 1984). Few markers have been shown to specifically demarcate the shell, but it is enriched in neurons expressing calbindin 28k (Grubb and Thompson, 2004). The terminals of Brn3a RGCs are highly enriched in the shell of the LGN, demarcating a previously unrecognized regional specificity of a class of contralateral projections to this structure in rodents. It is well established in several species that the eye-specific lamination of the ipsilateral projection to the LGN is dependent on spontaneous retinal depolarization, which occurs during the first postnatal week in mice (Shatz and Stryker, 1988; Feller, 2002; Huberman et al., 2003). It will be interesting to see whether the projections of the Brn3a RGCs also exhibit activity-dependent laminar specificity.

In contrast to the role of the DLG in cortical vision, the principal connections of the VLG and IGL are subcortical, and they mediate visuomotor and circadian functions (Harrington, 1997). The VLG and IGL are essentially devoid of Brn3a RGC axons, although some fibers appear to terminate in a very superficial zone of the VLG, immediately adjacent to the optic tract. Although attempts have been made to divide the VLG into "external" and "internal" domains (Swanson et al., 1974), it is not clear that any previous scheme identifies the very restricted area in which the Brn3a RGC fibers terminate or that any specific function has been assigned to this region.

Distal to the lateral geniculate, a complement of retinofugal axons leave the optic tract to innervate the pretectum, including the nucleus of the optic tract and the anterior, posterior, and olivary pretectal nuclei (Scalia and Arango, 1979). Functionally related to the accessory optic system, the pretectal nuclei serve subcortical visuomotor functions, including the pupillary light reflex, optokinetic responses, and visuovestibular integration (Pak et al., 1987). In the pretectal area, Brn3a RGC axons remain in the retinocollicular pathway and do not appear to terminate in any of the pretectal nuclei.

Finally, axons of Brn3a RGCs extensively innervate the superior colliculus. The uniform expression of Brn3a across the nasotemporal and dorsoventral retinal axes makes it unlikely that Brn3a plays a role in establishment of the retinocollicular topographic map. Consistent with this, the expression patterns of EphB1 and EphB3, which have been show to regulate retinocollicular maps (McLaughlin et al., 2003), are unaltered in the Brn3a knock-out retina. As expected, Brn3a RGC fibers appear to terminate uniformly throughout the superior colliculus, without a detectable mediolateral or rostrocaudal gradient, effectively ruling out a role for Brn3a in retinocollicular patterning.

In summary, these data indicate that Brn3a RGCs contribute only to the principal thalamocollicular visual pathway, mediating cortical vision, and are excluded from the subcortical pathways of the accessory optic system, pretectum, VGL, IGL, and hypothalamus, which mediate oculomotor reflexes and circadian rhythms. Thus, Brn3a is the first molecular/developmental marker for a large class of RGCs contributing specifically to cortical versus subcortical visual pathways.

In these experiments, determination of the precise role of Brn3a in RGC axon guidance decisions is limited by the neonatal death of Brn3a null mice and by probable redundancy among the Brn3 class of transcription factors expressed in the retina. Brn3a RGCs differentiate $\sim 2$ developmental days after the first detectable postmitotic retinal neurons, and likewise their axons are not the first to reach the optic chiasm and thalamic target fields. At E18.5, the latest day at which Brn3a null embryos could be examined with anterograde tracing, few Brn3a RGC fibers have penetrated the DLG, and essentially none have entered the superior colliculus, so the effect of the loss of Brn3a on the innervation of these structures could not be assessed.

Three members of the POU4 class of transcription factors, Brn3a, Brn3b, and Brn3c, are expressed in postmitotic RGCs, suggesting the possibility of partially overlapping function (Xiang et al., 1995). Mice lacking Brn3b exhibit a marked loss of RGCs and profound defects in retinal axon growth, with only a small fraction of RGC axons extending beyond the optic chiasm (Gan et al., 1999; Erkman et al., 2000). No specific retinal phenotype has been described previously for Brn3a or Brn3c null mutants, and the ganglion cell layer appears normal in number and 


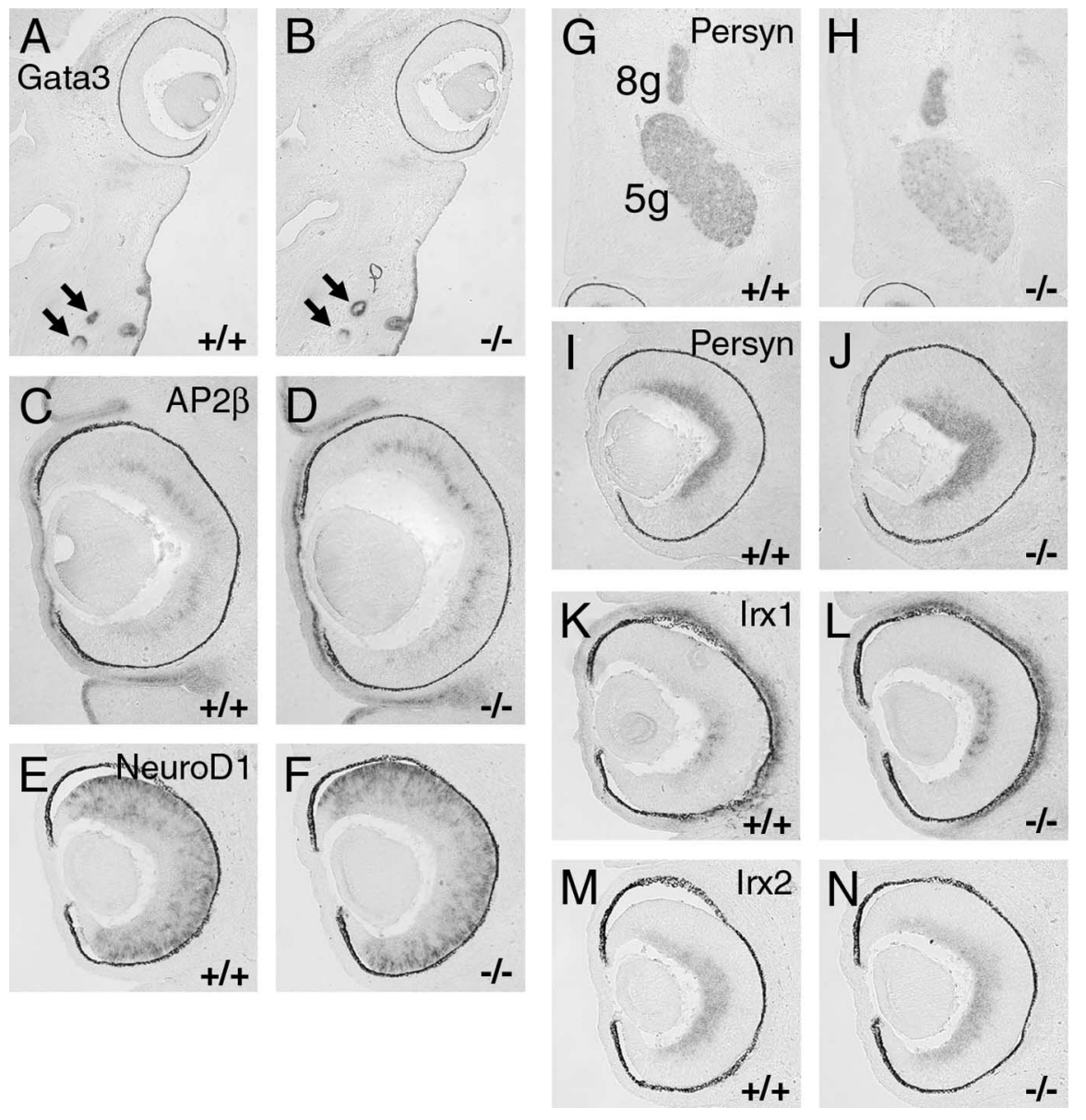

Figure 8. Expression of known Brn3a and Brn3b regulatory targets in the Brn3a ${ }^{-/-}$retina examined by in situ hybridization. Horizontal sections of E14.5 embryos are shown in all views, and the caudal/occipital part of the head or temporal side of the retina is at the top of each view. $A, B, G A T A 3$ is expressed strongly in whisker follicles (arrows) but not in the control or knock-out retina. $\mathbf{C}-\boldsymbol{F}$, Targets of Brn3a regulation in the trigeminal ganglion AP2 $\beta$ and NeuroD1 are unaltered in the Brn3a knock-out retina. $\mathbf{G}-\mathbf{J}$, Brn3b regulatory target persyn is decreased in the trigeminal ganglion but not in the retina of Brn3a null mice. $\boldsymbol{K}-\boldsymbol{N}$, Retinal expression of Irx1 is increased in the absence of Brn3a, but Irx2 shows no significant change. $5 \mathrm{~g}$, Trigeminal ganglion; $8 \mathrm{~g}$, vestibulocochlear ganglion.

morphology in mice lacking these factors (Xiang et al., 1996). However, loss of Brn3c does increase the severity of the Brn3b null phenotype (Wang et al., 2002). Possible factors contributing to the relative severity of the Brn3b null phenotype include the earlier developmental expression of Brn3b and the associated loss of Brn3a expression in Brn3b knock-outs (Gan et al., 1996; Xiang et al., 1997). It is unlikely that differences in protein function account for the distinct Brn3a and Brn3b phenotypes, because recent experiments have shown that the targeted replacement of Brn3b coding sequence with that of Brn3a rescues the Brn3b null phenotype (Pan et al., 2005).

In prenatal development, nearly all Brn3a RGCs coexpress at least low levels of Brn3b, suggesting that Brn3b significantly compensates for the loss of Brn3a in the embryonic retina. In other regions of the nervous system, such as the medial habenula, red nucleus, and peripheral sensory ganglia, where Brn3b is either not coexpressed or follows Brn3a in development, the effects of Brn3a null mutations are much more profound (Turner et al., 1994; Xiang et al., 1996; Eng et al., 2001). The extent of retinal coexpression of Brn3a and Brn3b appears to decline with advancing developmental age (Fig. 1), suggesting that a specific role for Brn3a may emerge in postnatal development. However, in the present study, the perinatal death of the Brn3a null mice prevents examination of the postnatal retina, and further understanding of the role of Brn3a in retinal development will require tissuespecific deletion of Brn3a to prevent the lethal effects of lesions in other parts of the nervous system.

\section{References}

Buchman VL, Adu J, Pinon LG, Ninkina NN, Davies AM (1998) Persyn, a member of the synuclein family, influences neurofilament network integrity. Nat Neurosci 1:101-103.

Eng S, Gratwick K, Rhee J, Fedtsova N, Gan L, Turner E (2001) Defects in sensory axon growth precede neuronal death in Brn3adeficient mice. J Neurosci 21:541-549.

Eng SR, Lanier J, Fedtsova N, Turner EE (2004) Coordinated regulation of gene expression by Brn3a in developing sensory ganglia. Development 131:3859-3870.

Erkman L, Yates PA, McLaughlin T, McEvilly RJ, Whisenhunt T, O'Connell SM, Krones AI, Kirby MA, Rapaport DH, Bermingham JR, O'Leary DD, Rosenfeld MG (2000) A POU domain transcription factor-dependent program regulates axon pathfinding in the vertebrate visual system. Neuron 28:779-792.

Fedtsova N, Turner E (1995) Brn-3.0 Expression identifies early post-mitotic CNS neurons and sensory neural precursors. Mech Dev 53:291-304.

Feller MB (2002) The role of nAChR-mediated spontaneous retinal activity in visual system development. J Neurobiol 53:556-567.

Gan L, Xiang M, Zhou L, Wagner DS, Klein WH, Nathans J (1996) POU-domain factor Brn-3b is required for the development of a large set of retinal ganglion cells. Proc Natl Acad Sci USA 93:3920-3925.

Gan L, Wang SW, Huang Z, Klein WH (1999) POU domain factor Brn-3b is essential for retinal ganglion cell differentiation and survival but not for initial cell fate specification. Dev Biol 210:469-480.

Godement P, Salaun J, Imbert M (1984) Prenatal and postnatal development of retinogeniculate and retinocollicular projections in the mouse. J Comp Neurol 230:552-575.

Grubb MS, Thompson ID (2004) Biochemical and anatomical subdivision of the dorsal lateral geniculate nucleus in normal mice and in mice lacking the beta2 subunit of the nicotinic acetylcholine receptor. Vision Res 44:3365-3376.

Harrington ME (1997) The ventral lateral geniculate nucleus and the intergeniculate leaflet: interrelated structures in the visual and circadian systems. Neurosci Biobehav Rev 21:705-727.

Hattar S, Liao HW, Takao M, Berson DM, Yau KW (2002) Melanopsincontaining retinal ganglion cells: architecture, projections, and intrinsic photosensitivity. Science 295:1065-1070.

Herrera E, Brown L, Aruga J, Rachel RA, Dolen G, Mikoshiba K, Brown S, Mason CA (2003) Zic2 patterns binocular vision by specifying the uncrossed retinal projection. Cell 114:545-557.

Huberman AD, Wang GY, Liets LC, Collins OA, Chapman B, Chalupa LM (2003) Eye-specific retinogeniculate segregation independent of normal neuronal activity. Science 300:994-998.

McEvilly RJ, Erkman L, Luo L, Sawchenko PE, Ryan AF, Rosenfeld MG (1996) Requirement for Brn-3.0 in differentiation and survival of sensory and motor neurons. Nature 384:574-577.

McLaughlin T, Hindges R, O'Leary DD (2003) Regulation of axial patterning of the retina and its topographic mapping in the brain. Curr Opin Neurobiol 13:57-69.

Mombaerts P, Wang F, Dulac C, Chao SK, Nemes A, Mendelsohn M, Ed- 
mondson J, Axel R (1996) Visualizing an olfactory sensory map. Cell $87: 675-686$.

Mu X, Beremand PD, Zhao S, Pershad R, Sun H, Scarpa A, Liang S, Thomas TL, Klein WH (2004) Discrete gene sets depend on POU domain transcription factor Brn3b/Brn-3.2/POU4f2 for their expression in the mouse embryonic retina. Development 131:1197-1210.

O'Gorman S, Dagenais NA, Qian M, Marchuk Y (1997) Protamine-Cre recombinase transgenes efficiently recombine target sequences in the male germ line of mice, but not in embryonic stem cells. Proc Natl Acad Sci USA 94:14602-14607.

Pak MW, Giolli RA, Pinto LH, Mangini NJ, Gregory KM, Vanable Jr JW (1987) Retinopretectal and accessory optic projections of normal mice and the OKN-defective mutant mice beige, beige-J, and pearl. J Comp Neurol 258:435-446.

Pak W, Hindges R, Lim YS, Pfaff SL, O'Leary DD (2004) Magnitude of binocular vision controlled by islet-2 repression of a genetic program that specifies laterality of retinal axon pathfinding. Cell 119:567-578.

Pan L, Yang Z, Feng L, Gan L (2005) Functional equivalence of Brn3 POUdomain transcription factors in mouse retinal neurogenesis. Development 132:703-712.

Paxinos G, Franklin KBJ (2001) The mouse brain in stereotaxic coordinates, Ed 2. San Diego: Academic.

Pennesi ME, Cho JH, Yang Z, Wu SH, Zhang J, Wu SM, Tsai MJ (2003) BETA2/NeuroD1 null mice: a new model for transcription factordependent photoreceptor degeneration. J Neurosci 23:453-461.

Reese BE (1988) 'Hidden lamination' in the dorsal lateral geniculate nucleus: the functional organization of this thalamic region in the rat. Brain Res 472:119-137.

Sanes JR, Yamagata M (1999) Formation of lamina-specific synaptic connections. Curr Opin Neurobiol 9:79-87.

Scalia F, Arango V (1979) Topographic organization of the projections of the retina to the pretectal region in the rat. J Comp Neurol 186:271-292.
Shatz CJ, Stryker MP (1988) Prenatal tetrodotoxin infusion blocks segregation of retinogeniculate afferents. Science 242:87-89.

Simpson JI (1984) The accessory optic system. Annu Rev Neurosci 7:13-41. Swanson LW, Cowan WM, Jones EG (1974) An autoradiographic study of the efferent connections of the ventral lateral geniculate nucleus in the albino rat and the cat. J Comp Neurol 156:143-163.

Trieu M, Rhee J, Fedtsova N, Turner E (1999) Autoregulatory sequences are revealed by complex stability screening of the mouse brn-3.0 locus. J Neurosci 19:6549-6558.

Trieu M, Ma A, Eng SR, Fedtsova N, Turner EE (2003) Direct autoregulation and gene dosage compensation by POU-domain transcription factor Brn3a. Development 130:111-121.

Turner E, Jenne K, Rosenfeld M (1994) Brn-3.2: a POU-domain transcription factor with distinctive CNS expression and regulation by retinoic acid. Neuron 12:205-218.

Wang SW, Mu X, Bowers WJ, Kim DS, Plas DJ, Crair MC, Federoff HJ, Gan L, Klein WH (2002) Brn3b/Brn3c double knockout mice reveal an unsuspected role for Brn3c in retinal ganglion cell axon outgrowth. Development 129:467-477.

Williams SE, Mason CA, Herrera E (2004) The optic chiasm as a midline choice point. Curr Opin Neurobiol 14:51-60.

Xiang M, Zhou L, Macke J, Yoshioka T, Hendry S, Eddy R, Shows T, Nathans J (1995) The Brn-3 family of POU-domain factors: primary structure, binding specificity, and expression in subsets of retinal ganglion cells and somatosensory neurons. J Neurosci 15:4762-4785.

Xiang M, Lin G, Zhou L, Klein WH, Nathans J (1996) Targeted deletion of the mouse POU-domain gene Brn-3a causes a selective loss of neurons in the brainstem and trigeminal ganglion, uncoordinated limb movement, and impaired suckling. Proc Natl Acad Sci USA 93:11950-11955.

Xiang M, Gan L, Li D, Chen ZY, Zhou L, O’Malley Jr BW, Klein W, Nathans J (1997) Essential role of POU-domain factor Brn-3c in auditory and vestibular hair cell development. Proc Natl Acad Sci USA 94:9445-9450. 